\title{
MAIANTHEMUM COMALTEPECENSE (CONVALLARIACEAE), UNA NUEVA ESPECIE DEL ESTADO DE OAXACA, MEXICO ${ }^{1}$
}

\author{
Adolfo Espejo Serna \\ Ana Rosa Lopez-Ferrari \\ JACQUeLINE CEJA ROMERO \\ Herbario Metropolitano \\ Departamento de Biología, C.B.S. \\ Universidad Autónoma Metropolitana \\ Unidad Iztapalapa \\ Apartado Postal 55-535 \\ 09340 México, D. F. \\ e-mail: aes@xanum.uam.mx
}

\begin{abstract}
RESUMEN
Se describe e ilustra Maianthemum comaltepecense Espejo, López-Ferrari \& Ceja, que se conoce hasta ahora de una sola población en el municipio de Santiago Comaltepec, distrito de Ixtlán de Juárez, Oaxaca, México. M. comaltepecense se distingue del resto de las especies mexicanas por su tamaño, por el número de hojas y por las características del rizoma y del fruto. Se incluye una clave de identificación, un mapa de distribución conocida, así como una relación de material colectado, correspondiente a cinco especies de Maianthemum registradas de Oaxaca.
\end{abstract}

\section{ABSTRACT}

Maianthemum comaltepecense Espejo, López-Ferrari \& Ceja is described and illustrated. It is presently known from a single population in the Municipio of Santiago Comaltepec, Distrito of Ixtlán de Juárez, Oaxaca, México. M. comaltepecense is distinguished from the other Mexican species by its size, number of leaves, and rhizome and fruit characteristics. For the five species of Maianthemum, reported from the state of Oaxaca, a key for identification, a distribution map and a list of collected material is included.

\section{INTRODUCCION}

El género Maianthemum comprende dieciséis especies americanas (Galway, 1944; Emons, 1945; LaFrankie, 1986; García-Arévalo, 1992), de las cuales diez se conocían para México (Espejo y López-Ferrari, 1995). Del estado de Oaxaca, se tenía registro solamente de dos: M. paniculatum (M. Martens \& Galeotti) LaFrankie y M. scilloideum (M. Martens \& Galeotti) LaFrankie (Espejo y López-Ferrari, 1995; López-Ferrari y Espejo, 1993). Como

\footnotetext{
${ }^{1}$ Trabajo parcialmente apoyado por la Comisión Nacional para el Conocimiento y Uso de la Biodiversidad a través del proyecto P-015.
} 
resultado de un viaje de exploración a la mencionada entidad, se recolectó material de una especie de Maianthemum y al tratar de identificarla se observó que no coincidía con las características de ninguno de los taxa conocidos para el país. La revisión de la literatura pertinente y del material del género depositado en los herbarios ENCB, MEXU y UAMIZ nos permite concluir que en Oaxaca crecen cinco especies: $M$. amoenum (H. L. Wendl.) LaFrankie, M. comaltepecense, M. macrophyllum (M. Martens \& Galeotti) LaFrankie, M. paniculatum y $M$. scilloideum. El material recientemente recolectado representa una novedad para la ciencia, por lo cual proponemos:

Maianthemum comaltepecense Espejo, López-Ferrari \& Ceja, sp nov. Fig. 1.

Herba terrestris, usque ad $30 \mathrm{~cm}$ alta. Rhizoma elongatum, cylindricum, $5-8 \mathrm{~cm}$ longum, 2-3 mm diametro. Radicibus solum ad nodos. Caules recurvati, flexuosi ad partem apicalem, glabri, $10-30 \mathrm{~cm}$ longi, 1-2 mm diametro. Folia 4-5(6), sessilia vel subsessilia, glabra, elliptica vel lanceolata, atro-viridia, $3-8.5 \mathrm{~cm}$ longa, $1-3 \mathrm{~cm}$ lata; Inflorescentia racemosa, $1.5-4.5 \mathrm{~cm}$ longa, $1-2$ floribus per nodum. Flores trimeri, non visi. Fructus trilobulatus, purpureus, albo-maculatus, 6-9 mm diametro. Semina lutea vel fusca, ca. $3 \mathrm{~mm}$ diametro.

Hierbas terrestres de 11 a $30 \mathrm{~cm}$ de largo. Unidades del rizoma alargadas, cilíndricas, pardo rojizas, de 5 a $8 \mathrm{~cm}$ de largo por 2 a $3 \mathrm{~mm}$ de diámetro; catáfilas papiráceas, translúcidas, presentes sólo en las unidades jóvenes del rizoma. Raíces únicamente en los nudos. Tallos recurvados, flexuosos en la parte apical donde llevan las hojas, glabros, de 10 a $30 \mathrm{~cm}$ de largo, por 1 a $2 \mathrm{~mm}$ de diámetro, los entrenudos de 0.7 a $3.4 \mathrm{~cm}$ de largo. Hojas 4 a 5(6), glabras, verdes obscuras, elípticas a lanceoladas, redondeadas en la base, agudas, con el margen escabriúsculo, de 3 a $8.5 \mathrm{~cm}$ de largo por 1 a $3 \mathrm{~cm}$ de ancho, sésiles a subsésiles. Inflorescencia un racimo compuesto, flexuoso, de 1.5 a 4.5 $\mathrm{cm}$ de largo, el raquis escabriúsculo, con 4 a 12 flores. Flores 1 a 2 por nudo, trímeras, no vistas. Fruto una baya trilocular, trilobada, de 6 a $9 \mathrm{~mm}$ de diámetro, de color púrpura con manchas blancas. Semillas 1 a 6, 2 por lóculo, globosas a levemente aplanadas, amarillas a pardas, ruguladas, de ca. $3 \mathrm{~mm}$ de diámetro.

TIPO: México, Oaxaca, distrito de Ixtlán de Juárez, municipio de Santiago Comaltepec, Cerro Pelón, ca. 4 km (en línea recta) al NE de Santiago Comaltepec, 17³4'40.8" N; 96³0'24" W, 2875-2900 m s.n.m., bosque de encino muy húmedo y umbrío, con abundantes musgos, líquenes y helechos, 10.IX.1995, A. R. López-Ferrari, A. Espejo y J. Ceja 2125 (Holótipo. UAMIZ 39319; isótipos (por distribuir): ENCB, GH, K, OAX).

Distribución: La nueva especie se conoce únicamente de una población en la localidad tipo, en bosques de encino neblinosos, húmedos y umbríos a una altura cercana a los 2900 m s.n.m.

Etimología: el epíteto específico hace referencia a Santiago Comaltepec, municipio en el cual fue encontrada la nueva especie.

Al utilizar la clave proporcionada por LaFrankie (1986 p. 388), M. comaltepecense quedaría incluida en el grupo de las especies norteamericanas de flores dímeras y hojas 
Espejo et al.: Maianthemum comaltepecense, Una Nueva Especie del Estado de Oaxaca

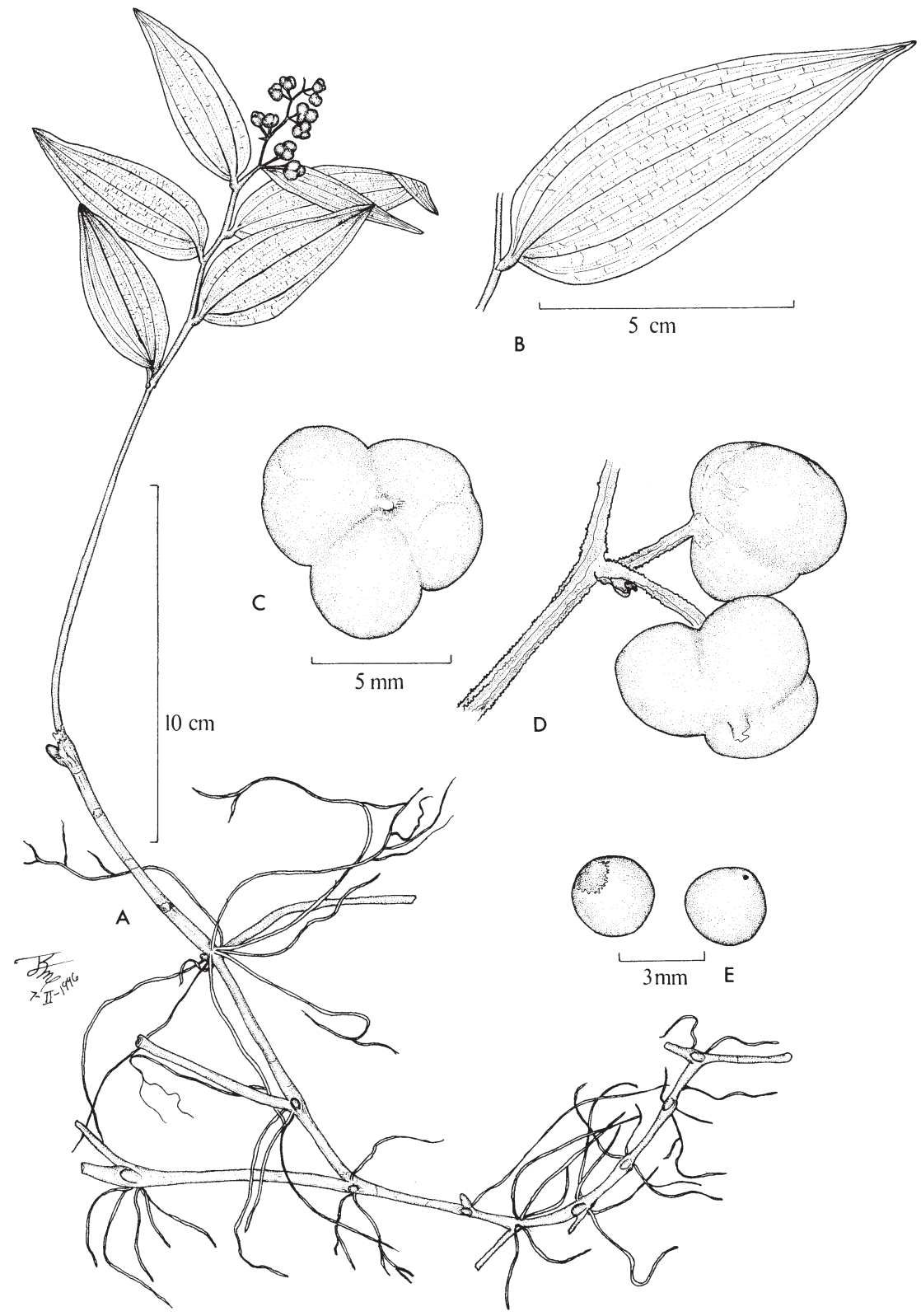

Fig. 1. Maianthemum comaltepecense Espejo, López-Ferrari \& Ceja. A. Hábito de la planta; B. Detalle de una hoja; C y D. Frutos; E. Semillas. Basado en el ejemplar A. R. López-Ferrari, A. Espejo y J. Ceja 2125. 
cordiformes ( $M$. canadense Desf. y $M$. dilatatum (Alph. Wood) Nelson \& MacBr.), por tener las unidades del rizoma cilíndricas y de menos de $3 \mathrm{~mm}$ de diámetro, las raíces restringidas a los nudos, las hojas en número reducido (2-6) y el fruto de color purpúreo con manchas blancas. Sin embargo, las flores trímeras y las hojas redondeadas en la base la distinguen de éstas sin lugar a dudas.

Por lo que toca a los representantes mexicanos del género, M. comaltepecense podría confundirse con $M$. scilloideum, especie cuya localidad tipo se encuentra también en Oaxaca (Cerro San Felipe, V-1839, H. Galeotti 5471), ya que ambas presentan rizomas cilíndricos y flores trímeras dispuestas en racimos compuestos con 1 a 2 flores por nudo. Sin embargo, las plantas de $M$. scilloideum son más robustas, portan raíces a lo largo de todo el rizoma, tienen generalmente 8 a 12 hojas por tallo y los frutos son rojos en la madurez y por lo regular esféricos (LaFrankie, 1986).

Con base en la revisión de los exsiccata depositados en los herbarios ENCB, MEXU y UAMIZ (Apéndice 1) se elaboró el mapa (Fig. 2) en el que puede observarse la distribución conocida de las cinco especies de Maianthemum que se presentan en el estado de Oaxaca. M. amoenum, M. macrophyllum y $M$. paniculatum se localizan en la vertiente atlántica de la Sierra Madre de Oaxaca (Sierra Mazateca y Sierra de Juárez) y $M$. macrophyllum se

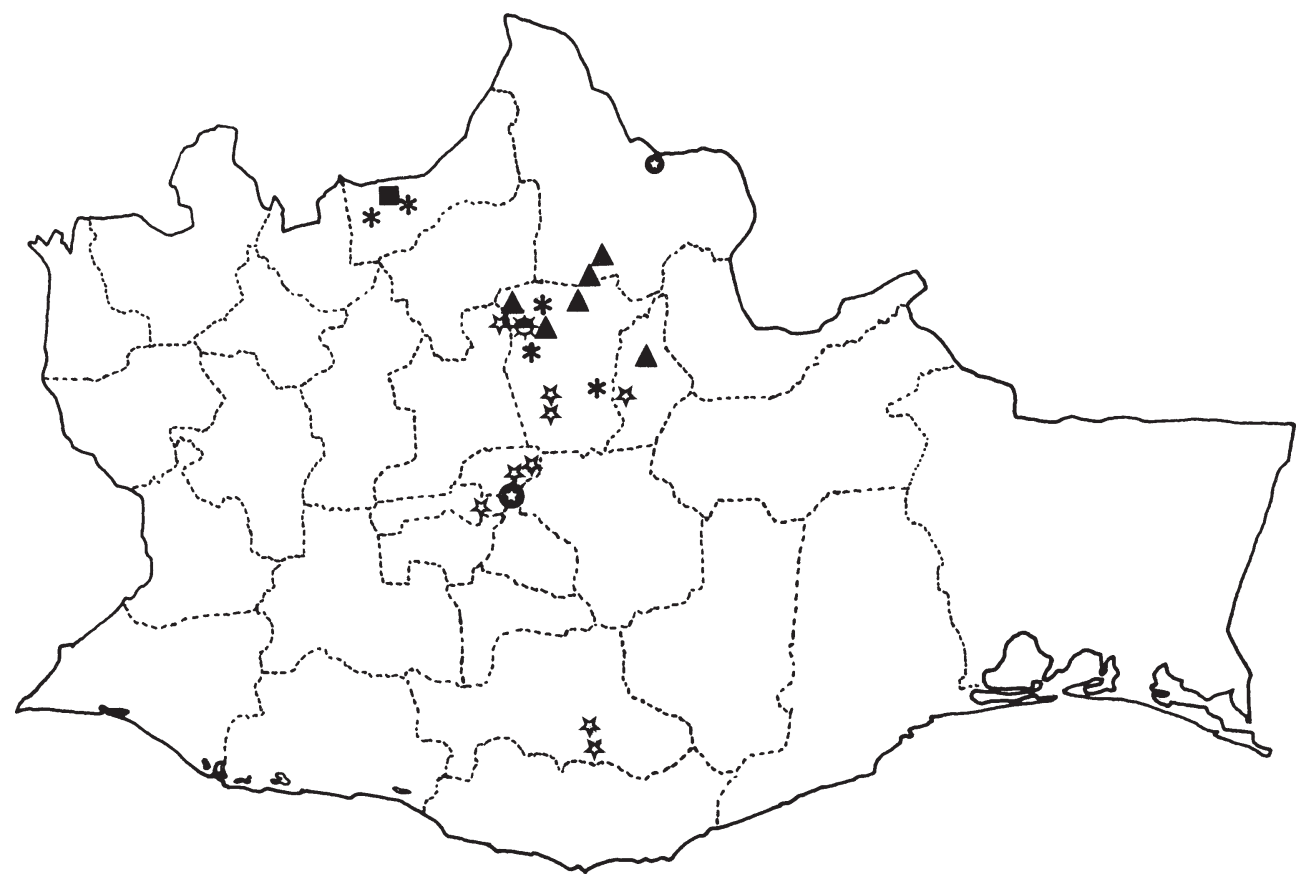

Fig. 2. Mapa que muestra la distribución conocida de las especies de Maianthemum que crecen en Oaxaca. M. amoenum * M. comaltepecense M. macrophyllum M. paniculatum $\triangle M$. scilloideum is 
registra por primera vez para la entidad, ya que dicha especie se consideraba endémica a Veracruz. M. scilloideum se presenta en la vertiente pacífica de la Sierra Madre de Oaxaca y $M$. comaltepecense se conoce únicamente de la localidad tipo en la parte más alta de dicha sierra.

A continuación se presenta una clave para identificar las especies de Maianthemum que crecen en Oaxaca.

1 Inflorescencia una panícula, los ejes laterales evidentes.

2 Pedicelos (0.6)1-2 cm de largo; ejes laterales basales de la inflorescencia fuertemente ascendentes, corimbiformes; perianto cupuliforme; plantas epífitas

M. amoenum

2 Pedicelos 0.2-0.8 cm de largo; ejes laterales basales de la inflorescencia extendidos o levemente ascendentes, racemiformes; perianto extendido; plantas generalmente terrestres M. paniculatum

1 Inflorescencia un racimo compuesto (1-2 flores por nudo).

3 Plantas epífitas, de 65 a $160 \mathrm{~cm}$ de alto; inflorescencia con 40 a 120 flores; fruto con 6 a 12 semillas M. macrophyllum

3 Plantas terrestres, de 11 a $50 \mathrm{~cm}$ de alto; inflorescencia con 4 a 35 flores; fruto con 1 a 6 semillas.

4 Hojas 6 a 11 por tallo; fruto maduro generalmente globoso, a veces levemente trilobulado, rojo; unidades individuales del rizoma 5-10 cm de largo por 3-10 $\mathrm{mm}$ de diámetro; raíces a todo lo largo del rizoma; semillas 1 por lóculo, 1 a 3 por fruto M. scilloideum

4 Hojas 4 a 5(6) por tallo; fruto maduro claramente trilobulado, purpúreo con manchas blancas; unidades individuales del rizoma 5-8 $\mathrm{cm}$ de largo por 2$3 \mathrm{~mm}$ de diámetro; raíces restringidas a los nudos; semillas 2 por lóculo, 1 a 6 por fruto M. comaltepecense

\section{AGRADECIMIENTOS}

Queremos hacer patente nuestro agradecimiento a los Drs. Victoria Sosa y Ramón Riba por la revisión crítica del manuscrito y sus valiosas sugerencias al mismo. El dibujo fue elaborado por el Biól. Rolando Jiménez Machorro. Finalmente agradecemos a los curadores de los herbarios consultados por las facilidades otorgadas.

\section{LITERATURA CITADA}

Emons, R. W. 1945. A revision of the Central American species of Smilacina. Ann. Missouri Bot. Gard. 32: 395-411.

Espejo, A. y A. R. López-Ferrari. 1995. Las monocotiledóneas mexicanas, una sinopsis florística. 1. Lista de Referencia, parte IV. Commelinaceae, Convallariaceae, Costaceae, Cyclanthaceae y Cymodoceaceae. Consejo Nacional de la Flora de México, A. C., Universidad Autónoma Metropolitana y Comisión Nacional para el Conocimiento y Uso de la Biodiversidad. México, D.F. 53 pp.

Galway, D. H. 1944. The North American species of Smilacina. Amer. Midl. Naturalist 33: 644-666. 
Acta Botánica Mexicana (1996), 36:21-28

García Arévalo, A. 1992. Maianthemum mexicanum (Liliaceae), una nueva especie de Durango, México. Acta Bot. Mex. 17: 19-21.

LaFrankie Jr., J. V. 1986. Morphology and taxonomy of the New World species of Maianthemum (Liliaceae). J. Arnold Arbor. 67: 371-439.

López-Ferrari, A. R. y A. Espejo. 1993. Convallariaceae. In: Flora de Veracruz 76: 1-20. 
Espejo et al.: Maianthemum comaltepecense, Una Nueva Especie del Estado de Oaxaca

\section{APENDICE 1}

\section{Maianthemum amoenum}

Dto. de Ixtlán, $19 \mathrm{~km}$ al $\mathrm{N}$ de Llano de las Flores, carretera a Valle Nacional, $R$. Torres C. y D. Lorence 285 (ENCB, MEXU); Distr. Mixe, desviación a Tiltepec, $1 \mathrm{~km}$ al NW de la desviación a San Andrés Yaa, R. Torres C., R. Cedillo y L. Rico 471 (MEXU); Distrito de Teotitlán, en el Puerto de la Soledad, a $30 \mathrm{~km}$ al S de Huautla sobre el camino a Teotitlán, O. Téllez y W. Elisens 4693 (MEXU); $60 \mathrm{~km}$ al N de Ixtlán, sobre la carretera a Tuxtepec, J. Rzedowski 30623 (ENCB); Puerto de la Soledad, Teotitlán del Camino, T. MacDougall H338 (ENCB).

\section{Maianthemum macrophyllum}

Mpio. Teotitlán de Flores Magón, 8 km al NW de Huautla de Jiménez, hacia Teotitlán del Camino, Rafael Torres C. y M. Antonio Martínez 6561 (MEXU).

\section{Maianthemum paniculatum}

Mpio. Ixtlán de Juárez, 3 km adelante de La Esperanza, camino Oaxaca-Tuxtepec, A. R. López Ferrari y A. Espejo 701 (MEXU, UAMIZ); Distrito de Ixtlán, $13 \mathrm{~km}$ al N de La Esperanza, Abisaí García M., R. Torres y L. Cortés 3025 (MEXU); Distrito de Ixtlán, ruta 175 Tuxtepec a Oaxaca, cerca Vista Hermosa (a km 80), David H. Lorence y Refugio Cedillo Trigos 3142 (MEXU); Distrito Ixtlán, a $31 \mathrm{~km}$ al S de Valle Nacional, camino a Oaxaca, E. Martínez S., O. Téllez, G. Davidse y J. Davidse 8764 (MEXU); a 10 km del Cerro "Pelado", camino a Tuxtepec, Emma Saavedra 7(7) (MEXU); Brecha 200 desviación antigua al Rancho La Primavera, terrenos de Macuiltianguis, Enrique Guízar N. 778 (UAMIZ); Distrito de Ixtlán, entre Vistahermosa y Comaltepec, km 82 carretera Oaxaca-Tuxtepec, G. Martínez Calderón 252 (ENCB); Mpio. Santiago Comaltepec, Distrito de Ixtlán, Puerto Eligio, km 149 carretera Oaxaca-Tuxtepec, G. Martínez Calderón 774 (MEXU, UAMIZ); Distrito de Ixtlán, Vistahermosa, Comaltepec, km 135 carretera Oaxaca-Tuxtepec, G. Martínez Calderón 820 (MEXU); Paraje de "Yaa chulla", arriba de La Primavera, Macuiltianguis, Sierra de Juárez, Guillermo Pérez Cruz C 124 (ENCB, MEXU); San Juan Bautista Valle Nacional, a 1 km antes de La Esperanza, carretera de Valle Nacional a Cerro Pelón, J. I. Calzada, J. Paniagua y V. López 16690 (MEXU); Sierra San Pedro Nolasco, cerca de Talea de Castro, Ladislao Paray 579 (ENCB); Sierra de Juárez, Norma Spíndola 22 (MEXU); Mpio. Santiago Comaltepec, Puerto Antonio, $5.3 \mathrm{~km}$ al N de Vista Hermosa, carretera Oaxaca-Tuxtepec, 17³8' N 96²2' W, Pedro Tenorio L. y R. Torres C. 11044 (MEXU); km 94 carretera Tuxtepec-Valle Nacional, R. Riba y G. Pompa R-5803 (MEXU); Distr. de Ixtlán, 24.8 km al N de Humo Chico, carretera Oaxaca-Tuxtepec, R. Torres C. y R. Cedillo T. 1449 (MEXU); Mpio. Santiago Comaltepec, Dto. Ixtlán, 22 km al O de La Esperanza, carretera TuxtepecOaxaca, Rafael Torres C., A. García M. y L. Cortés 9555 (MEXU); Mpio. Santiago Comaltepec, Dto. Ixtlán, $5 \mathrm{~km}$ al O de La Esperanza, carretera Tuxtepec-Oaxaca, 17³6' N 96²4' W, Rafael Torres C. y E. Martínez S. 11328 (MEXU); Mpio. Santiago Comaltepec, Dto. Ixtlán, Vista Hermosa, $26.6 \mathrm{~km}$ al SO de Valle Nacional, carretera Tuxtepec-Oaxaca, 17043' N 96²0' W, Rafael Torres C. y E. Martínez S. 11406 (MEXU); Mpio. Santiago Comaltepec, Dto. Ixtlán, Vista Hermosa, $26.6 \mathrm{~km}$ al SO de Valle Nacional, carretera Tuxtepec-Oaxaca, 17043' N 96²0' W, Rafael Torres C. y E. Martínez S. 11421 (MEXU); 
Mpio. Santiago Comaltepec, Dto. Ixtlán, Vista Hermosa, 26.6 km al SO de Valle Nacional, carretera Tuxtepec-Oaxaca, 1743' N 96²0' W, Rafael Torres C. y E. Martínez S. 11423 (MEXU); Mpio. Santiago Comaltepec, Distr. Ixtlán, Vista Hermosa, 28 km al S de Valle Nacional, carretera Tuxtepec-Oaxaca, Rafael Torres C. y L. Cortés 8670 (MEXU); Mpio. Santiago Comaltepec, Distr. Ixtlán, $11.4 \mathrm{~km}$ al S de La Esperanza, carretera TuxtepecOaxaca, Rafael Torres C. y L. Cortés 8733 (MEXU); Mpio. Santiago Comaltepec, Dto. Ixtlán, Puerto Antonio, $5 \mathrm{~km}$ al $\mathrm{N}$ de Vista Hermosa, carretera Tuxtepec-Oaxaca, Rafael Torres C. y L. Cortés 8753 (MEXU); Mpio. Santiago Comaltepec, Dto. Ixtlán, entrada a San Isidro Yólox, 10.9 km al SO de La Esperanza, carretera Tuxtepec-Oaxaca, 17³6' N 96²3' W, Rafael Torres C. y L. Cortés A. 11714 (MEXU); 4 km al SW de La Esperanza, Refugio Cedillo T., Rafael Torres y L. Rico 2416 (ENCB); East-facing slopes, mountains along Route 175, $28 \mathrm{~km}$ by road south Valle Nacional on the way to Ixtlán de Juárez, Robert Merrill King 2115 (MEXU); $60 \mathrm{~km}$ al N de Ixtlán, sobre la carretera a Tuxtepec, J. Rzedowski 30624 (ENCB); $39 \mathrm{~km}$ al S de Valle Nacional, sobre la carretera a Oaxaca, J. Rzedowski 33347 (ENCB); $39 \mathrm{~km}$ al S de Valle Nacional, sobre la carretera a Oaxaca, J. Rzedowski 33779 (ENCB); $39 \mathrm{~km}$ al S de Valle Nacional, sobre la carretera a Oaxaca, J. Rzedowski 33915 (ENCB); Mpio. Santiago Comaltepec, $82 \mathrm{~km}$ al SW de Tuxtepec, sobre la carretera a Oaxaca, Stephen D. Koch y T. R. Soderstrom 77250 (ENCB); Along highway 175 through Sierra de Juárez between Tuxtepec and Oaxaca, 18.4 miles south of bridge at Valle Nacional at ca. km 140, Thomas B. Croat 48046 (MEXU).

\section{Maianthemum scilloideum}

Sierra de San Felipe, $6.2 \mathrm{~km}$ después de La Cumbre, sobre la desviación a Corral de Piedra, 17011'30" N 96³7'30" W, A. Espejo y A. R. López Ferrari 5047 (UAMIZ); Mpio. Tonaltepec, $4 \mathrm{~km}$ adelante de "La Cumbre", rumbo a Ixtlán de Juárez, A. Espejo, A. $R$. López Ferrari, M. Flores C. y J. Santana 4065 (UAMIZ); Mpio. Suchixtepec, $5 \mathrm{~km}$ adelante de San José del Pacífico, rumbo a Puerto Angel, A. R. López Ferrari y A. Espejo 1020 (UAMIZ); Vicinity of Cerro Zempoaltépetl, East slopes at Patio de Arena about five kilometers east of summit, Boone Hallberg 820 (MEXU); Cerro San Felipe, C. Conzatti y V. G. 704 (MEXU); Sierra de San Felipe, C. G. Pringle 4647 (ENCB, MEXU); Mpio. Santiago Comaltepec, Distrito de Ixtlán, $1 \mathrm{~km} \mathrm{~N}$ of Comaltepec on path to Yolox, Gary J. Martin 541 (ENCB); Mountains along highway 175, about 12-13 km north of Ixtlán de Juárez, road to Tuxtepec, Gordon D. McPherson 826 (ENCB); Campamento Río de Molino, 4 km al SW de San Miguel Suchixtepec, J. Rzedowski 21080 (ENCB); 27 km N of Ixtlán at km 85 on road to Tuxtepec, upper slopes of Sierra Juárez, 17030' N 963' W, Keith Roe y Eunice Roe 1964 (ENCB); Mpio. Ixtlán de Juárez, 14 km al N de Ixtlán, R. Fernández N. 2408 (ENCB); San Pablo Cuatro Venados, Dto. Zaachila, 4 km sobre la desviación a Contreras Peniles, carretera a San Miguel Peras. Reg. Valles Centrales, R. López Gómez 293 (UAMIZ). 